\title{
The Development of Interior Decoration Design Under the Background of Big Data
}

\author{
Yin Wenjing ${ }^{1}$ \\ ${ }^{1}$ Wuhan Institute of Shipbuilding Technology,Wuhan,China,430050.
}

\begin{abstract}
In recent years, with the rapid development of information technology and the extensive coverage of the Internet, the information age has come, and data is gradually becoming one of the important factors in the production of various manufacturing and operational entrepreneurs. With the development of society, people have higher and higher requirements for indoor planning. People no longer only pursue the beauty of a simple natural environment, but also pursue the beauty of the natural environment, their unique personality and spiritual pursuit. This article firstly studies some development issues and trends of interior decoration design, and then discovers the application of big data in interior decoration design through literature research. Finally, the questionnaire surveyed the interior decoration design style and product selection. The survey results showed that about $50 \%$ of the choice of style chose simple style, and $70 \%-80 \%$ of consumers chose natural materials for decoration products. It can be seen that, the interior decoration design is simpler, and the natural style develops.
\end{abstract}

\section{Introduction}

Big data, the Internet of Things and cloud computing have become hot topics today, and are gradually entering all aspects of life [1-2]. Under the thinking of the big data era, everything can be "quantified" and the world is made up of information. According to this big data function [3-4], it is extended to interdisciplinary interior decoration design, which generates new design ideas and directions, and promotes the new development of interior decoration design [5-6]. In the era of big data, the research and development of interior decoration design [7-8].

In the research on the combination of computer multimedia technology and English teaching, many scholars have studied it and achieved good results. For example, J Sun has passed relevant research on information quality control and major CBR process models, and has also studied user behavior in depth. Pattern, the formation mechanism of user behavior patterns. Discussed in detail the interior decoration design ideas based on CBR and the user behavior mode of information quality control [9]. Obermeyer $\mathrm{Z}$ by focusing on the relationship between interaction design and big data, some reasonable suggestions were put forward [10].

This article firstly studies some development issues and trends of interior decoration design, and then discovers the application of big data in interior decoration design through literature research. Finally, the questionnaire surveyed the interior decoration design style and product selection. The survey results showed that about $50 \%$ of the choice of style chose simple style, and $70 \%-80 \%$ of consumers chose natural materials for decoration products. It can be seen that, The interior decoration design is simpler, and the natural style develops.

\section{Research on interior decoration design}

\subsection{Research Methods}

\subsubsection{Literature research:}

Reading books and articles about interior decoration design and big data related literature at home and abroad, the advantage is that you can understand the development process of the research object from the source, and understand the development status of the research object, and provide a clear and structured theoretical basis for indepth thesis development.

\subsubsection{Investigation and research method}

The questionnaire survey method is that this article conducts a survey through pre-prepared questions and analyzes the answers of the interviewees to draw the necessary conclusions. Through the design of questionnaires, to understand the changes in the learning situation of the research objects, the purpose of which is mainly to investigate students' interest, views and feelings about the ecologicalization of college English classrooms. 


\subsubsection{Quantitative analysis}

Qualitative analysis is related to quantitative analysis. Quantitative analysis refers to the analysis of mathematical hypothesis determination, data collection, analysis and testing, and it is also quantitative and qualitative.

Qualitative analysis refers to the qualitative analysis of the research object. It refers to the process of conducting research based on subjective understanding and qualitative analysis, through research and bibliographic analysis.

\subsection{Problems in Interior Decoration Design}

\subsubsection{Consumer psychology}

Just like "there are thousands of villages in the eyes of thousands of people", different consumers and users have different aesthetics and consumption abilities, so they also have different requirements for interior design. Moreover, the different habits, hobbies, and economic conditions of consumers and users make interior decoration design neglected by consumers.

\subsubsection{Lack of professional interior decoration designers}

In our country, not many companies specialize in interior decoration design. Most of them are concentrated in firstclass cities. The visibility of second- and third-tier cities is very low. Therefore, the limited demand on the market made it impossible to specialize in soft decoration from the beginning. Most people are interior designers at first, and then gradually come into contact with interior decoration design to understand soft design. This type of conversion takes a long time to learn and practice the knowledge of upholstered furniture, and the process is very long.

\subsection{Application of Big Data in Interior Decoration Design}

\subsubsection{Artificial intelligence}

Human-computer interaction and intelligent processing are no longer new topics. In fact, these two technologies have been reflected in our lives for a long time. The emergence of intelligent technology enables computers to process human-like thoughts, and to process voice, images, and video. In addition, with the help of artificial intelligence, we can also monitor and perceive the environment and information of the house in real time. Through the Internet, users can even remotely track family information. Therefore, artificial intelligence not only provides us with a more comfortable lifestyle, but artificial intelligence will become the main trend of future social development.

\subsubsection{Dataization}

In this era of big data, how to effectively use information technology to optimize and process information has become an urgent problem to be solved. In fact, interior decoration designers can make full use of public data to create greater effects for them. By automatically creating and managing the interior design database, controlling and completing the data required for the design work, the authenticity and convenience of interior design can be improved and supplemented.

\subsubsection{Diversification}

Diversity combines intelligence, communication, human culture and ecology at the same time. In order to meet the different housing needs of different users, it is necessary to provide users with different housing experiences in different forms. For example, while providing people with a harmonious and natural ecological home, it also temporarily introduced future technical design. Make full use of big data technology, computer modeling, IBM, VR and other technologies to simulate and simulate the space design.

\subsubsection{Personalization}

Humanitarian care and its personalized design methods will become the development and main offensive direction of the interior design field in the future. More and more high-quality employees and young people are looking for their own personalized life style and services. Only by providing these customers with personalized products and design services, can they themselves be favored and satisfied by these customers in the market, which is conducive to the economic and social development of our country. In fact, the level of design will directly affect the satisfaction and comfort of customers' daily life.

\subsection{Development Trend of Interior Decoration Design}

\subsubsection{Human culture of interior decoration design}

With the globalization of the economy, the continuous prosperity and development of my country's market economy has led to the improvement of people's living standards. People have begun to pursue spiritual enjoyment and hope to make their living space cultural and intellectual. Adopt to change the interior, color, material, cold and heat, light, etc. make indoor culture and emotions.

\subsubsection{Humanization of interior decoration design}

Living in a residential area "factory facing", many houses have similar layouts, so some decoration companies only use patterns for decoration. More and more people are tired of it and have the same decoration methods and styles. Not suitable for users with different needs. Users need special plans for their houses, and designers need to design 
humanized solutions suitable for their lives according to their own living habits. This makes it a soft decorative design, developing in the direction of humanization.

\subsubsection{Ecologicalization of interior decoration design}

With the deterioration of the ecological environment every year, more and more people are living in "reinforced concrete boxes", returning to nature, and the concept of protecting the environment is getting stronger and stronger. Natural styles will become more and more popular, and natural textured materials are gradually being widely used in various designs. Designers try to use natural materials to evoke memories of nature. Therefore, soft interior decoration will become more and more ecological.

\subsection{Calculation of Interior Decoration Design System}

\subsubsection{Min count algorithm}

The probability density function sets $\mathrm{x}$ as a random variable. If there is a non-negative real number $\mathrm{f}(\mathrm{x})$, so that for any real number $\mathrm{a}<\mathrm{b}$, there is $\mathrm{P}\{\mathrm{a} \leq \mathrm{x}<\mathrm{b}\}=\mathrm{ff}(\mathrm{x}) \mathrm{dx}$, then $\mathrm{X}$ is called continuous Type random variable, $\mathrm{f}(\mathrm{x})$ is the probability density function of $\mathrm{x}$. The Min Count algorithm was proposed in Reference 667]. The algorithm performs cardinality estimation based on the statistical information of the hash result. As the name implies, it is based on the minimum value of the sequence to estimate the cardinality. Assuming that the minimum value of the hash result of all elements in the multiset is $\mathrm{X}$, the algorithm approximates that the estimate of the set cardinality $n$ is one. The minimum probability density $f(x)$ of $\mathrm{n}$ random $\mathrm{X}$-machine uniform variables in the interval is $n(1-x) "-1$, so the mathematical expectation of the minimum value satisfies the following equation:

$$
\mathrm{E}(\mathrm{M})=\int_{0}^{1} x \cdot n(n-1)^{n-1} d x=\frac{1}{n+1} .
$$

According to the calculation result of the minimum mathematical expectation of the interval $[0,1]$, it is easy to think of: when one is expected, its value is approximately equal to $\mathrm{M}$. When the min Count algorithm is analyzed and studied mathematically, it is found that $\mathrm{x}=0$ is one. Mathematical expectation integral equation-a divergence point, the following formula:

$$
\mathrm{E}\left(\frac{1}{M}\right)=\int_{0}^{1} \frac{1}{x} \cdot n(1-x)^{n-1} d x=+\infty
$$

\subsubsection{LogLog Counting algorithm}

The main idea of the algorithm: For the data set $\mathrm{D}$, scan all the elements $\mathrm{x}$ in the set, and for each element $\mathrm{x}$ scanned, it is mapped into a binary bit string of $\{0,1\}$ through a hash function, denoted by $\mathrm{p}(\mathrm{x})$ The position where the first number "1" appears in the binary bit string, such as $\mathrm{p}(, \ldots, \mathrm{p} 0(00 .)=$.2 , etc., when all elements in the set are hashed, record the first hash result The maximum value of the position where "1" appears, expressed by pmx (x), can be considered as the base estimate:

$$
\mathrm{E}=2^{\rho_{\min }(x)}
$$

\section{Investigation of Interior Decoration Design}

\subsection{Research Purpose}

With the gradual improvement of people's quality of life, people pay more and more attention to their own living environment. With the advent of the era of big data, this article studies the status quo of interior decoration, and chooses the style of interior decoration design under the background of big data.

\subsection{Questionnaire Design}

(1) User's basic personal information, gender, age, income, occupation, etc. To complete the personal information needed to build the character, please make the character model closer to reality.

(2) The user's goals, motivations, attitudes and needs are the core of the questionnaire. Why do users choose interior decoration products, what goals do they want to achieve after use, etc.

(3) Use background. Use time and user interface information.

(4) Related parameters of usage behavior, such as usage frequency, usage intensity, etc. These data will help collect consumer perceptions of the product.

\subsection{Questionnaire Collection}

\subsubsection{Number of questionnaires}

According to the minimum sample size formula in statistics, the author sets the confidence level of the questionnaire to $80 \%$, and the allowable error does not exceed $8 \%$. Calculate the minimum sample size as

$$
n_{0}=\left(\frac{t_{a}}{2 \Delta p}\right)^{2}=\left(\frac{1.645}{2 \times 0.075}\right)^{2}=120
$$

That is, the minimum sample size of this questionnaire is 120 copies.

\subsubsection{Source of questionnaire}

There are a wide range of consumers of interior decoration design, and there are no big restrictions on age, gender and region, so there is no specific field of research topic. The way of distributing questionnaires is: online surveys are mainly conducted on the Star questionnaire website, followed by distribution of printed questionnaires and interviews between relatives and friends. Consumers of wearable products are very extensive, and there are no major restrictions on age, gender and region. Therefore, there is no specific area of research subject. The way to distribute the questionnaire is: mainly conduct online surveys on the star questionnaire website, and secondly distribute the printed questionnaires and conduct interviews between relatives and friends. 


\section{Data Analysis}

This article uses a questionnaire survey to study the styles of consumers wanting interior decoration design. The results of the survey are shown in Table 1.

Table 1. The style of interior decoration design

\begin{tabular}{|c|c|c|}
\hline & male & women \\
\hline minimalism & $48 \%$ & $50 \%$ \\
\hline Classical & $10 \%$ & $8 \%$ \\
\hline Japanese style & $30 \%$ & $32 \%$ \\
\hline European style & $12 \%$ & $10 \%$ \\
\hline
\end{tabular}

The style of interior decoration design

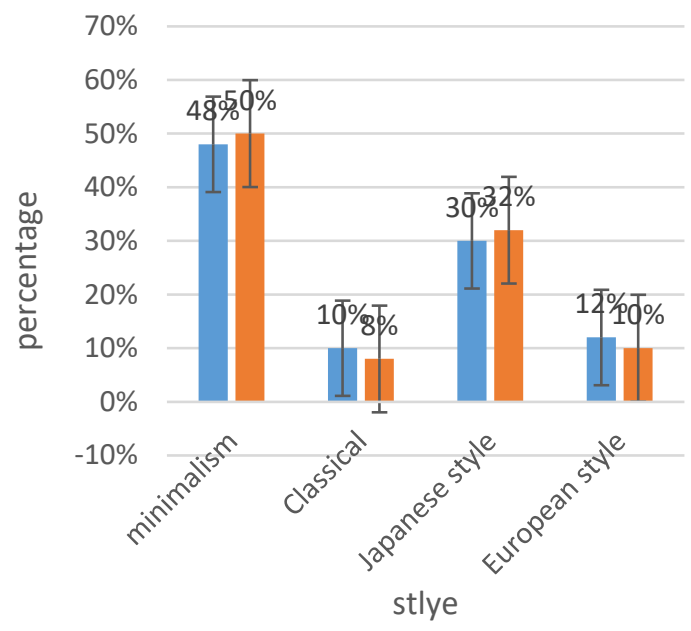

male women

Figure 1. The style of interior decoration design

As shown in Figure 1, the survey results show that about $50 \%$ of the choices of style choose simple style. With the advent of the era of big data, big data technology will make our living and living environment more concise. This is why most people choose simple interior decoration design.

This article uses a questionnaire to investigate the types of interior decoration design products, and the results of the survey are shown in Table 2.

Table 2.Types of interior decoration design products

\begin{tabular}{|c|c|c|c|}
\hline & youth & $\begin{array}{c}\text { middle } \\
\text { aged }\end{array}$ & elderly \\
\hline $\begin{array}{c}\text { Natural } \\
\text { Ingredients }\end{array}$ & $58 \%$ & $60 \%$ & $72 \%$ \\
\hline $\begin{array}{c}\text { Man-made } \\
\text { materials }\end{array}$ & $42 \%$ & $40 \%$ & $28 \%$ \\
\hline
\end{tabular}

Types of interior decoration design products

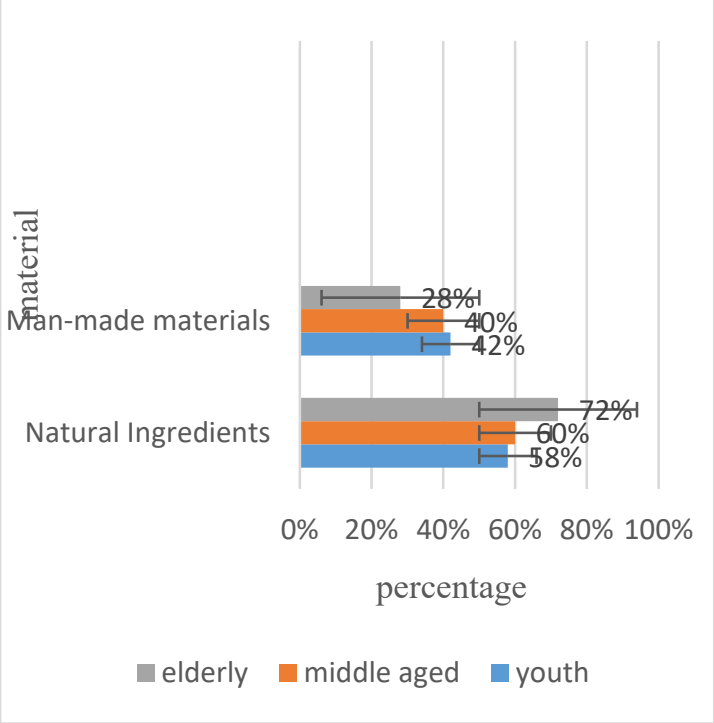

Figure 2. Types of interior decoration design products

It can be seen from Figure 2 that about 70\%-80\% choose natural materials. As people pay more attention to the natural environment, more and more people choose environmentally friendly products that are harmless to nature and harmless to human health.

\section{Conclusion}

Based on the relationship with big data, a huge network has been established between different disciplines to connect two previously unrelated things. This also requires interior decorators, not just designers. It not only pays attention to trends, but also involves various fields such as social policy, environment and humanities, and obtains information through a lot of observations to help us design. There is also the use of design-related ideas. In the past, it was very perceptual. The design conclusion was "to shoot the head". Using the dual combination of rationality and sensitivity in the design. The combination of big data and design can transform interior decoration design into more Sustainability and social market-oriented behavior. At the same time, there are more and more successful data-driven change cases, which are undoubtedly the demonstration, promotion and development of interior decoration design.

\section{References}

1. He J . Interior Decoration Design and Construction Technology of Hardcover Room[J]. Journal of Physics: Conference Series, 2020, 1649(1):012005 (5pp).

2. Li S, Hou Y. Study on the Architecture Design and interior Decoration based on VR Technology and Computer Simulation Platform[J]. Paper Asia, 2019, 35(2):54-57

3. Naimi A I, Westreich D J . Big data : a revolution that will transform how we live, work, and think | 
$\mathrm{Clc}[\mathrm{J}]$. American Journal of Epidemiology, 2014, 179(9):1143-1144..

4. Lynch, Clifford. Big data: How do your data grow?[J]. Nature, 2008, 455(7209):28-29.

5. D'Angeac G D . Big Data: The Management Revolution - Harvard Business Review[J]. Harvard Business Review, 2012, 90(10):60-6, 68, 128

6. Varian $H$ R . Big Data: New Tricks for Econometrics $[\mathrm{J}]$. Journal of Economic Perspectives, 2014, 28(2):3-27.

7. Ibrahim, Abaker, Targio, et al. The rise of "big data" on cloud computing: Review and open research issues[J]. Information Systems, 2015, 47(Jan.):98115.

8. Zaharia M, Xin R S, Wendell P, et al. Apache Spark: a unified engine for big data processing[J]. Communications of the Acm, 2016, 59(11):56-65..

9. Sun J, Li S, Yan N, et al. Research on User Behavior Patterns under the Background of Big Data[J]. Journal of Physics: Conference Series, 2020, 1639(1):012018 (5pp).

10. Obermeyer $Z$, Emanuel E J . Predicting the Future - Big Data, Machine Learning, and Clinical Medicine.[J]. N Engl J Med, 2016, 375(13):12161219. 